\title{
$\beta$-烯胺酯为关键中间体的串联反应研究进展
}

\author{
韩莹孙晶孙岩高红颜朝国* \\ (扬州大学化学化工学院 扬州 225002)
}

\begin{abstract}
摘要 串联反应具有原料简单易得、中间产物无需分离提纯、操作简单、节省溶剂和降低消耗等很多独特优点，已成 为当前有机合成研究的热点课题. 缺电子炔烃与芳香胺在温和条件下快速反应生成的 $\beta$-烯胺酯, 具有丰富的化学反应 性质，可以作为多组分串联反应的关键中间体，和其它组分进行串联反应，构建结构复杂的碳链、碳环、杂环化合物和 螺环化合物. 综述了近年来此类直接生成的 $\beta$-烯胺酯参与的多组分串联反应的最新研究进展.

关键词 串联反应; 多组分反应; 缺电子炔烃; 烯胺酯; 杂环化合物; 螺环化合物
\end{abstract}

\section{Development of Domino Reactions with $\beta$-Enamino Esters as Key Intermediates}

\author{
Han, Ying Sun, Jing Sun, Yan Gao, Hong Yan, Chaoguo* \\ (College of Chemistry \& Chemical Engineering, Yangzhou University, Yangzhou 225002)
}

\begin{abstract}
Domino reactions have attracted much high attention in recent years due to its convergence, productivity, facile execution and generation of highly diverse and complex products from easily available starting materials in a single operation. Addition of primary amines to electron-deficient alkynes could generated reactive enaminone (ester) in mild conditions, which have shown versatile reactivity and have been widely used in domino procedure as key reactive intermediate to develop a number of carbon-carbon bond formation reactions, heterocyclic and spiro cyclic constructions. In this paper the recent developments of the domino reactions with enamino esters as key intermediates based on our experimental investigation are reviewed.
\end{abstract}

Keywords domino reaction; multicomponent reaction; electron-deficient alkyne; enamino ester; heterocycles; spirocycles

绿色化学合成方法的研究是当前有机合成化学的 热点问题. 串联反应作为一种绿色化学合成方法近几年 得到了迅速发展 ${ }^{[1,2]}$. 串联反应通常指将多步反应或多 个反应组分置于一个反应器中完成, 中间产物不需加以 分离和提纯, 直接得到最终产物. 它具有反应条件温 和、原料简单易得、中间体无需分离，操作简单、节省 溶剂、降低消耗等独特的优点 ${ }^{[3 \sim 6]}$. 因此在有机合成中 有着广泛的应用, 也是当前有机合成研究的热点问题.

缺电子炔烃与氮杂芳烃形成 1,4-偶极中间体 (Scheme 1)的反应非常容易, 在温和条件下就能快速完 成. Huisgen 等 ${ }^{[1]}$ 最先认识到 $1,4-$ 偶极中间体和常见的 1,3-偶极中间体具有相同的电子结构, 可以进行环加成 反应，形成含氮六元杂环化合物. 近年来人们还发现，
丁炔二酸二甲酯、丙炔酸甲酯、乙酰基乙炔和苯甲酰基 乙炔等常用的缺电子炔烃和亲核试剂如氮杂芳烃、有机 膦、异氰酸酯等反应形成的 1,4-偶极中间体具有丰富的 反应活性 ${ }^{[2]}$, 并且此类 1,4-偶极中间体无需事先制备, 可以现场生成后再和其它含 $\mathrm{C}=\mathrm{C}, \mathrm{C}=\mathrm{O}, \mathrm{C}=\mathrm{N}$ 等双键 型化合物进行串联加成、环化等反应，形成众多的杂环 化合物体系(Scheme 2) $)^{[3 \sim 7]}$.

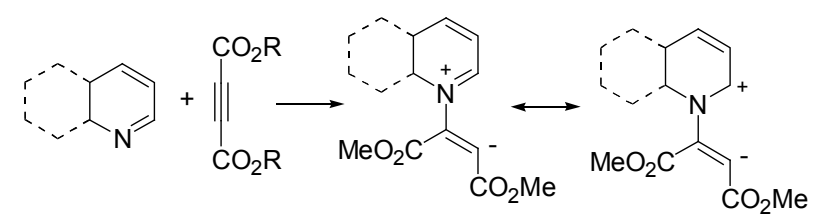

Scheme 1

\footnotetext{
* E-mail: cgyan@yzu.edu.cn

Received June 8, 2012; revised July 12, 2012; published online July 17, 2012.

Project supported by the National Natural Science Foundation of China (Nos. 20972132, 21172189).

国家自然科学基金(Nos. 20972132, 21172189)资助项目.
} 


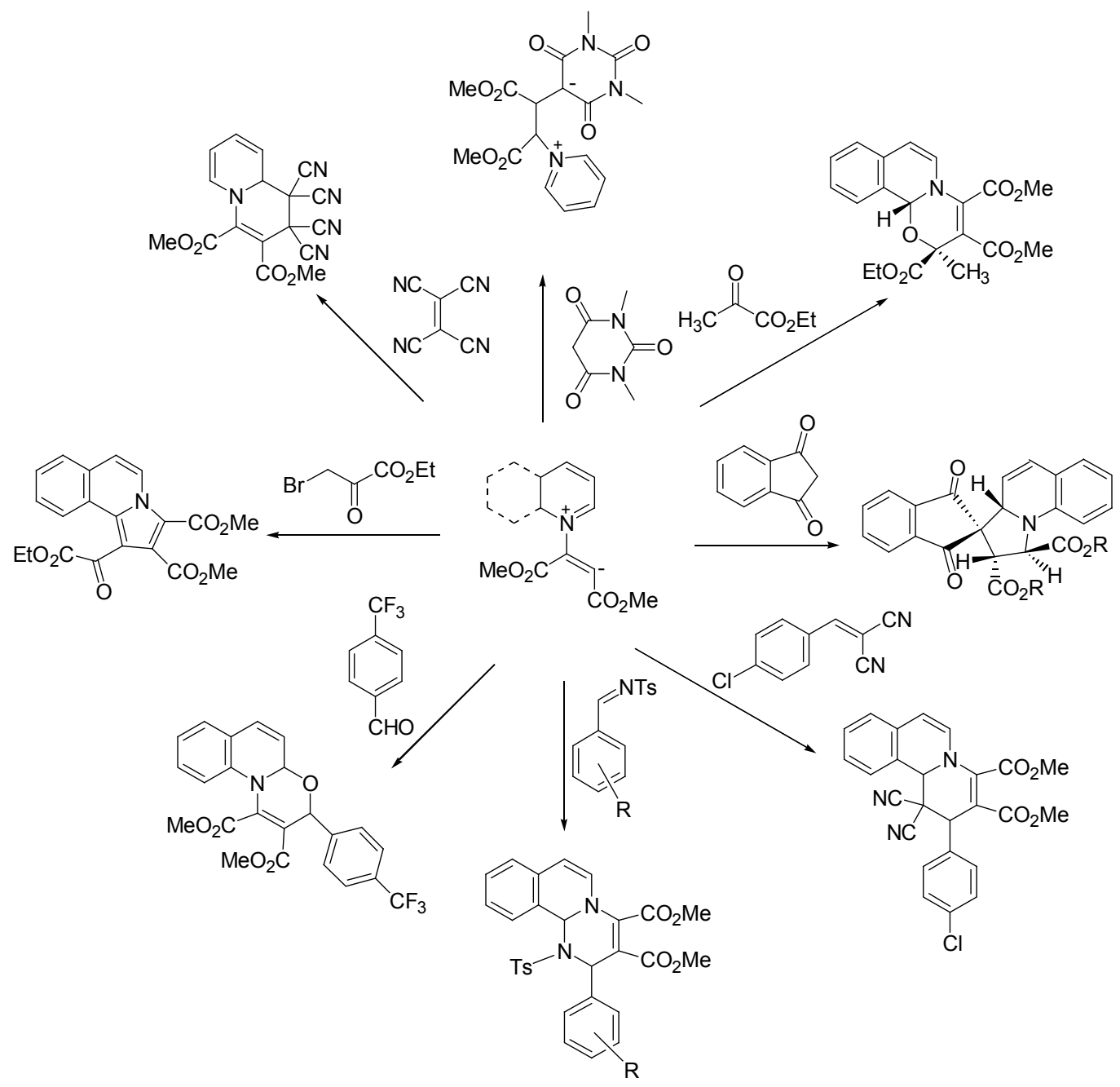

Scheme 2

缺电子炔烃与伯胺或仲胺在有机溶剂中也可以快 速进行加成反应，生成比较稳定的烯胺酮(酯)(Eq. 1), 所得烯胺酮(酯)的顺反异构体比例和反应条件有关, 特 别是溶剂的性质对其影响较大 ${ }^{[8]}$. 由于此反应为可逆反 应，在溶液中顺反异构体可以相互转化 ${ }^{[9]}$.

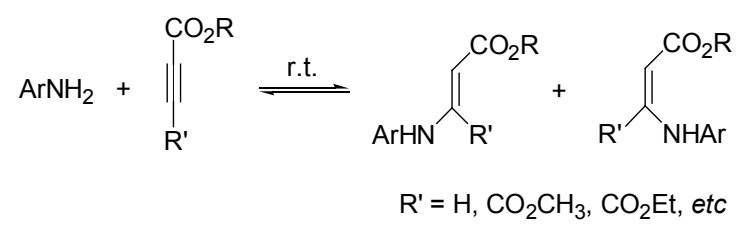

$\beta$-烯胺酮(酯)是一类结构和性质都比较特别的化合 物, 分子中既含有强亲核性的烯胺结构单元, 又含有亲 电性的烯酮结构单元, 还存在着互变异构现象, 整个分 子具有多个活性反应中心, 分子中 C-1 和 C-3 为缺电子 中心, 而 C-2 和氨基是富电子中心, 具有丰富的化学反 应性质, 是一种重要的有机合成子. $\beta$-烯胺酮(酯)可以和 $\alpha, \beta$-不饱和化合物发生亲核加成、环合、胺解和脱氢等 串联反应, 生成含氮五元或六元杂环化合物; 也可以作
为亲二烯体发生共轭加成反应; 同时烯胺酮(酯)也是一 种 1,3-偶极中间体，可以和各种活泼双键化合物进行 1,3-偶极环加成反应 ${ }^{[10 ~ 12] . ~} \beta$-烯胺酮(酯)的制备方法很 多，最直接的方法是通过胺和 $\beta$-二羰基化合物的缩合反 应制备，但一般都需要预先制备，才能应用于后续反 应 ${ }^{[13]}$. 由脂肪族或芳香族伯胺和缺电子炔烃加成生成 $\beta$ 烯胺酩(酯)的反应可以在无催化剂和室温条件下，短时 间内完成. 因此可以通过胺和缺电子炔烃反应现场生成 烯胺酩(酯), 不用预先制备, 作为串联反应的关键合成 子，这就为设计多组分串联反应提供了理想的途 径 ${ }^{[14 ~ 16]}$. 已有作者介绍了部分缺电子炔烃和胺等亲核 试剂加成形成的中间体在有机合成中的应用情况 ${ }^{[17]}$, 本文结合我们在缺电子炔烃参与的多组分串联反应的 相关研究工作，介绍最新的研究进展.

\section{1 丁炔二酸二甲酯参与的四组分串联反应}

在丁炔二酸二甲酯、芳香胺、芳香醛和丙二腈的四 组分串联反应中, 先将芳香醛、丙二腈在三乙胺、乙醇 
体系中室温下反应 $10 \mathrm{~min}$, 然后加入芳胺与丁炔二酸二 甲酯, 继续反应 $12 \mathrm{~h}$ 得到了全取代 2-氨基-1,4-二氢吡啶 衍生物(Eq. 2). 在此四组分反应中, 芳香胺和芳香醛中 取代基的影响很小, 不论是强吸电子取代基还是强给电 子取代基，也不论取代基的位置是邻位、间位还是对位， 都以 $80 \% \sim 96 \%$ 的高产率得到了目标化合物 ${ }^{[18]}$.

$$
\mathrm{ArCHO}+\mathrm{CH}_{2}(\mathrm{CN})_{2}+\mathrm{Ar}^{\prime} \mathrm{NH}_{2}+\|\left.\right|_{\mathrm{CO}_{2} \mathrm{R}} ^{\mathrm{Ct}_{2} \mathrm{H}}
$$

在类似反应条件下, 如用氰乙酸乙酯、氰基频哪酠 替代丙二腈为反应底物, 该四组分串联反应也能顺利完 成，以 75\% 94\%的产率合成了全取代 2-氨基-1,4-二氢
吡啶衍生物. 但用氰乙酰胺替代丙二腈, 在室温条件下 反应很慢, 将温度提高到 $60{ }^{\circ} \mathrm{C}$, 可以顺利得到中等产 率的目标产物. 如果在反应中使用对苯二胺或 4,4'-联苯 二胺等芳香二胺为芳香胺组分，则可以得到芳基桥联的 双 1,4-二氢吡啶衍生物, 表明该四组分串联反应具有比 较广泛的适应性(Scheme 3).

很显然，该四组分串联反应是按照以下的串联反应 过程完成的(Scheme 4). 在反应过程中, 现场生成的烯 胺酯 $\mathbf{B}$ 和现场生成的芳亚甲基丙二腈 $\mathbf{A}$ 通过 Michael 加成形成中间体 $\mathbf{C}$ ，再通过分子内氨基对氧基的加成反 应生成环状中间体 $\mathbf{E}$ ，然后通过异构化完成反应，生成 二氢吡啶衍生物.

Prasad 等 ${ }^{[19]}$ 以三氯化铟为催化剂, 使用 3-氨基咔 唑、芳香醛、丙二腈、丁炔二酸酯或丙炔酸酯, 研究了 类似的四组分反应，得到了咔唑取代的 1,4-二氢吡啶衍 生物. 如果采用微波加热，该反应可以在 $10 \mathrm{~min}$ 内完成 (Eq. 3).

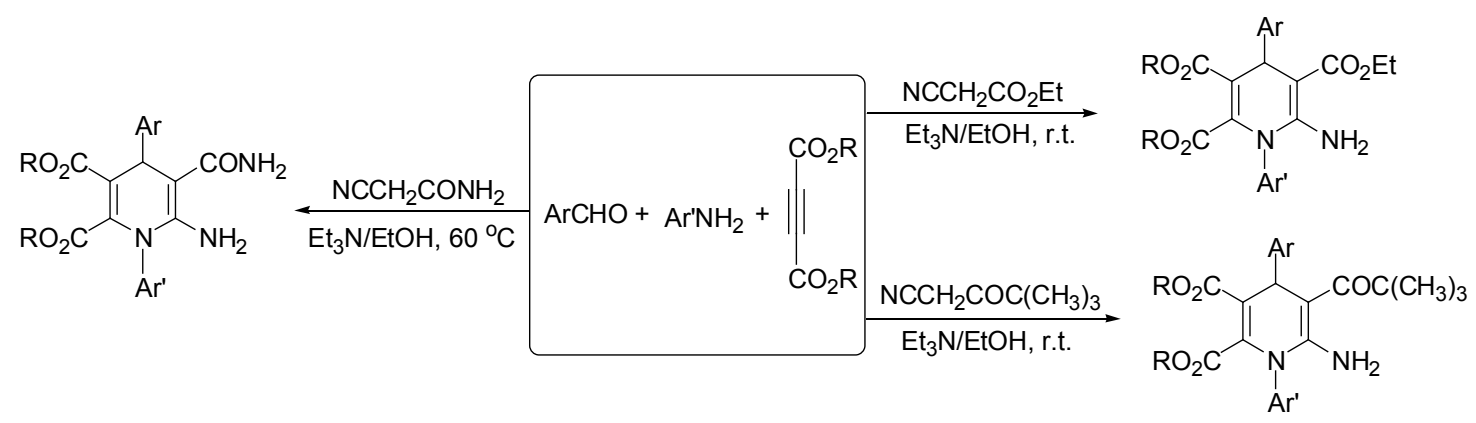

Scheme 3

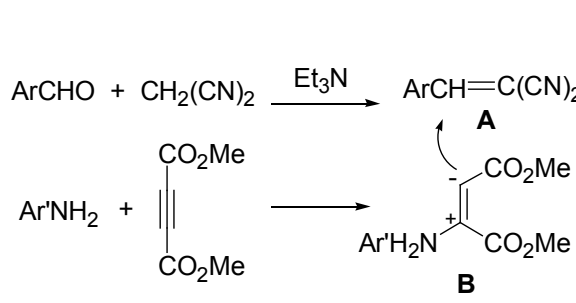

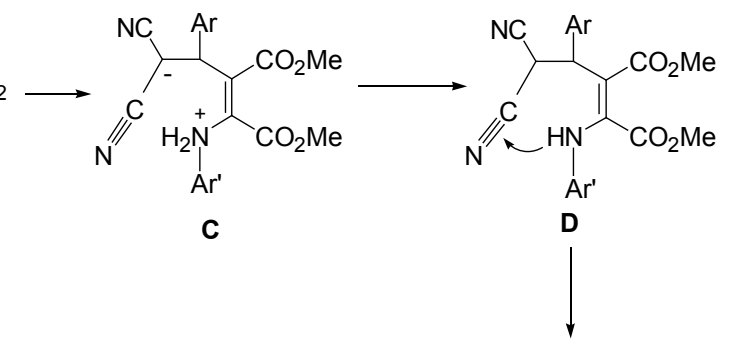<smiles>COC(=O)C1=C(C(=O)OC)N([Al])C(N)=C(C#N)C1Br</smiles><smiles>CC(=O)C1=C(C(C)=O)N([Ga])C(=N)C(C#N)C1Br</smiles>

Scheme 4

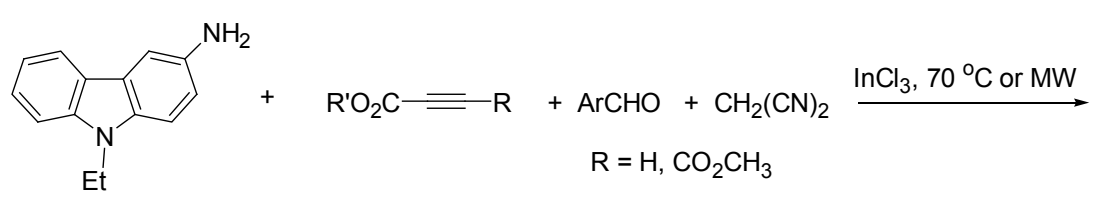<smiles>[R]OC(=O)C1=C([R])N(c2ccc3c(c2)c2ccccc2n3CC)C(N)=C(C#N)C1[Al]</smiles> 
最近，雷明等 ${ }^{[20]}$ 报道了一个很有创意的多组分反 应, 将肉桂醛亚胺、丁炔二酸酯和芳香醛在乙腈中进行 反应，生成较高产率的多取代 1,3-氧氮杂环衍生物(Eq. 4), 该反应不具备立体选择性, 得到的顺式产物比例稍 高于反式产物. 反应过程为肉桂醛亚胺和丁炔二酸酯加 成形成 1,4-偶极中间体, 后者与芳香醛基进行 [4+2]加 成反应.

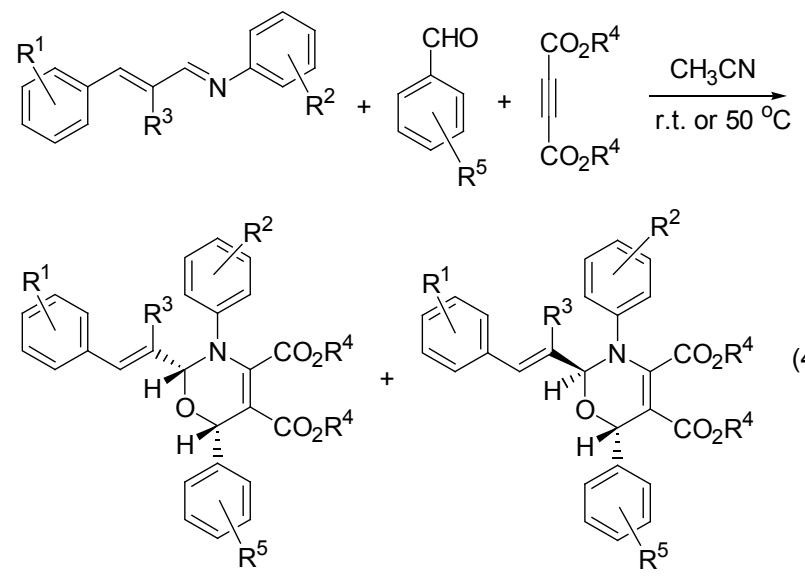

江焕峰等 ${ }^{[21]}$ 报道了丁炔二酸酯、两分子甲醛和两分 子胺进行的五组分反应, 生成了嘧啶衍生物. 在反应中 可以使用两种不同的胺, 根据加入的顺序, 得到不同的 产物(Eq. 5). 先加入的胺组分连接在丁炔二酸酯结构单 元上, 后加入的胺组分和甲醛结构单元相连. 这清楚表 明反应中胺和丁炔二酸酯形成了关键的 $\beta$-烯胺酯中间 体.

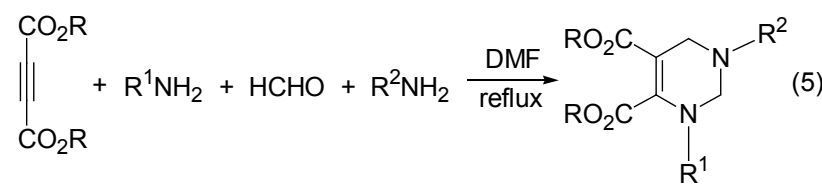

如果先将芳胺与丁炔二酸二甲酯在乙醇中进行加 成反应, 生成 $\beta$-烯胺酯, 再加入醛和 5,5-二甲基-1,3-环 己二酮组分 ${ }^{[22]}$, 在室温下反应, 发现芳胺中的氨基并没 有进行类似的串联反应生成吡啶环，而是 5,5-二甲基1,3-环已二酮分子中的一个羰基参与串联反应形成 3,4二氢吡喃衍生物, 产率在 $64 \% \sim 85 \%$ 之间. 得到的 3,4二氢吡喃衍生物核磁氢谱显示存在两种不同的异构体, 顺反异构体的比例一般在 $1: 5 \sim 1: 7$ 之间, 这明显是 由于芳胺与丁炔二酸二甲酯加成生成的 $\beta$-烯胺酯中间 体存在顺式与反式两种结构导致的, 当然环合过程的空 间效应也会影响产物的立体选择性. 此反应的机理与前 述反应相类似. 在反应过程中, 芳胺与丁炔二酸二甲酯 发生加成反应生成 $\beta$-烯胺酯, 芳醛与 5,5-二甲基-1,3-环 己二酮发生 Knoevenagel 缩合反应生成 $\alpha, \beta$-不饱和羰基
化合物, $\beta$-烯胺酯与 $\alpha, \beta$-不饱和羰基化合物发生 Micheal 加成反应，再通过烯醇负离子进攻双键环合形成含氧六 元环化合物(Eq. 6).
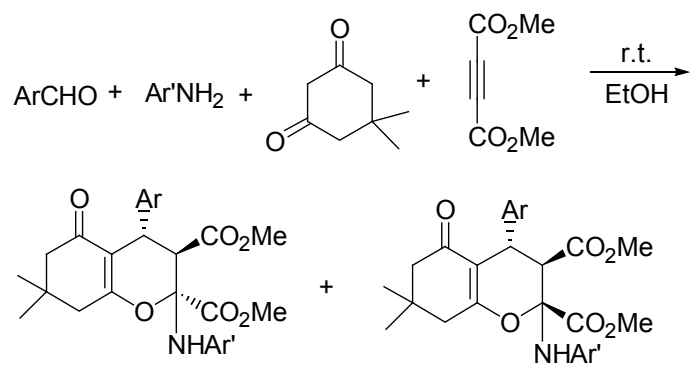

其它环状 1,3-二羰基化合物如 1,3-环已二酮、4-差 基香豆素也能进行上述反应，生成类似 3,4-二氢吡喃衍 生物. 如果在反应中使用丙二酸亚异丙酯作为环状 1,3二羰基化合物，则生成多取代吡啶-2-酮衍生物(Scheme $5)$, 但收率略有降低, 这是因为丙二酸亚异丙酯在反应 过程中容易发生开环裂解.

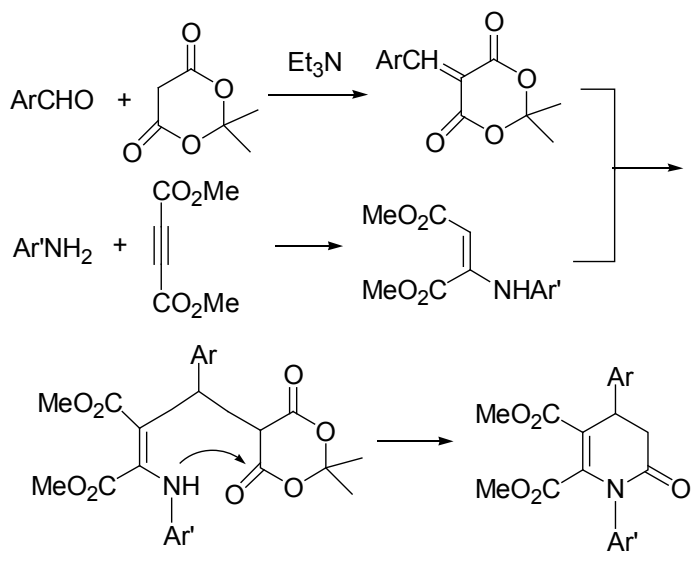

Scheme 5

在按照一般条件进行的芳胺、丁炔二酸二甲酯、芳 香醛和 1,3-环己二酮的四组分反应中，未能生成预期的 1,4-吡啶环类衍生物，而是意外地得到了 3,4-二氢吡喃 衍生物。但是，根据上述类似反应的机理，该反应体系 应该可以形成 1,4-二氢吡啶环类衍生物. 果然, 通过改 变反应条件, 在醋酸溶液中芳胺、丁炔二酸二甲酯形成 $\beta$-烯胺酯, 然后加入芳香醛和 1,3 -环己二酮， $80{ }^{\circ} \mathrm{C}$ 下反 应可以顺利地制备 1,4-二氢吡啶衍生物，收率在 $60 \%$ 左 右(Scheme 6) ${ }^{[23]}$. 5,5-二甲基-1,3-环已二酮和 1,3-环戊二 酮也能很好地参与反应，形成一系列 1,4-二氢吡啶衍生 物. 在个别反应中, 还发现了少量的 1,2-二氢吡啶衍生 物副产物生成. 观察 1,2-二氢吡啶衍生物的结构可以看 出, 其中没有芳胺和丁炔二酸二甲酯形成的 $\beta$-烯胺酯结 构单元，但具有芳香醛和芳香胺形成的亚胺结构单元. 这表明 1,2-二氢吡啶衍生物是按照不同的反应机制形成 
的，因此通过改变反应的操作步骤，将预先制备好的芳 香亚胺醋酸溶液加入到 1,3-环己二酮和丁炔二酸二甲酯 反应后的乙醇溶液中, 可以得到中等收率的 1,2 -二氢吡 啶衍生物. 这为难以合成的 1,2-二氢吡定衍生物提供了 一种非常简洁有效的合成方法.

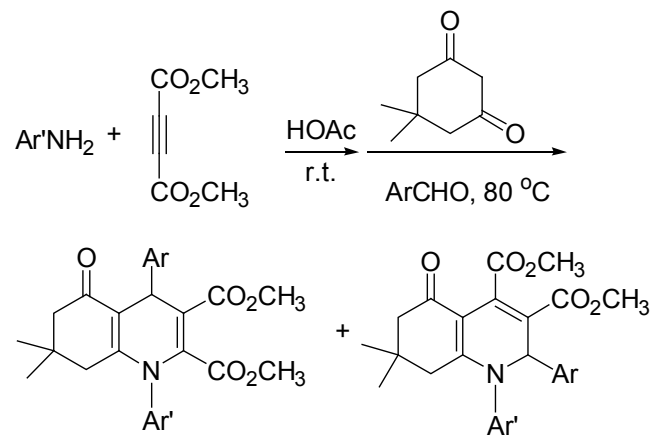<smiles>[R]C1CC(=O)CC([R])C1</smiles><smiles></smiles>

\section{Scheme 6}

由于靛红分子中的 3-位羰基具有较强的反应活性, 可以形成多种多样含有氧化吲哚的螺环化合物, 因此在 上述四组分串联反应中使用静红代替芳香醛参与反应, 即芳香胺、丁炔二酸二甲酯、靛红和 5,5-二甲基 1,3-环
己二酮四组分串联反应体系，在醋酸溶液中于室温下反 应, 得到了中等产率的螺 [1,2-二氢吡啶-2-氧化吲哚]衍 生物 ${ }^{[24]}$. 该反应条件温和, 操作方便, 一步反应就可以 得到复杂的螺环化合物. 但通过改变反应条件, 希望生 成螺 [1,2-二氢吡啶-2-氧化吲哚]的努力没有取得成功. 在类似反应条件下，1,3-环已二酮也能进行此类四组分 反应，生成中等以上产率的螺 [1,2-二氢吡啶-2-氧化吲 哚]衍生物。该四组分反应是通过以下机理完成的, 5,5二甲基 1,3-环已二酮和丁炔二酸二甲酯反应形成加成中 间体 $\mathbf{A}$, 芳香胺和靛红反应形成 3-芳基亚胺基靛红 $\mathbf{B}$, 两者通过加成反应形成新的中间体 $\mathbf{C}$, 再经过分子内的 脱水反应，形成目标产物(Scheme 7).

进一步的研究发现, 现场形成的 $\beta$-烯胺酯和靛红、 丙二腈的串联反应, 在三乙胺存在下可以高产率地生成 螺 $\left[1,4-\right.$ 二氢吡啶-2-氧化吲哚]衍生物(Eq. 7) ${ }^{[25]}$. 反应过 程也是经过 $\beta$-烯胺酯和在体系中生成的双氧基亚甲基 靛红的加成反应, 然后芳胺基对氧基进行分子内加成反 应完成的. Perumal 等 ${ }^{[26]}$ 同期开展了类似的研究工作.

很有意思的是芳香胺、丁炔二酸二甲酯、静红和丙 酮在醋酸中的四组分反应，形成了靛红取代的螺[吡咯 烷-3-酮-2-氧化吲哚]衍生物(Eq. 8), 其中缺少丁炔二酸 二甲酯结构单元，说明其没有参加反应 ${ }^{[27]}$. 如果将芳香 胺和丁炔二酸二甲酯在醋酸中预先反应形成 $\beta$-烯胺酯, 再加入靛红和丙酮, 反应的结果相同. 这是由于 $\beta$-烯胺 酯的生成反应是可逆平衡反应, 在该反应体系中分解成 丁炔二酸二甲酯和芳香胺所致.

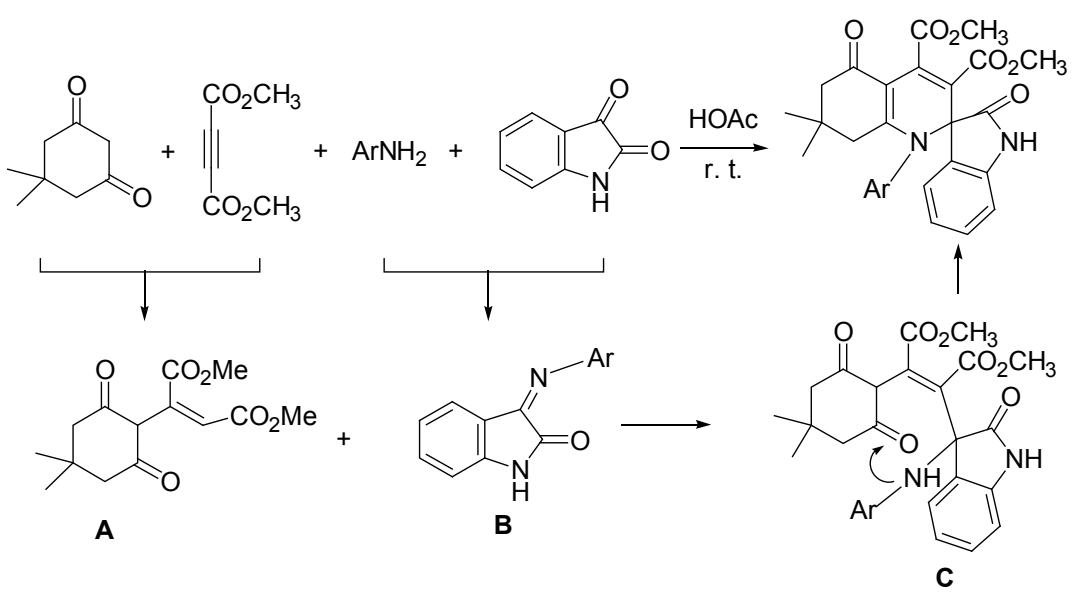

Scheme 7<smiles>[R]c1ccc2c(c1)C(=O)C(=O)N2[Z7](=O)[O-]</smiles> 
<smiles>[R]c1ccc2c(c1)C(=O)C(=O)N2</smiles><smiles>[R]c1ccc2c(c1)C(C1C(=O)CC3(C(=O)Nc4ccc([R])cc43)N1[O-])C(=O)N2</smiles>

Alizadeh 等 ${ }^{[28]}$ 报道了脂肪伯胺、丁炔二酸酯、丙二 腈和狮三酮在水中的四组分反应, 无需加入催化剂, 生 成了结构特殊的杂环 [3.3.3]螺旋桨烷衍生物(Heterocyclic[3.3.3]propellanes) (Eq. 9). 该反应经过了一系列复 杂的串联反应，但也包含了 $\beta$-烯胺酯的生成和对羰基的 加成反应等过程.

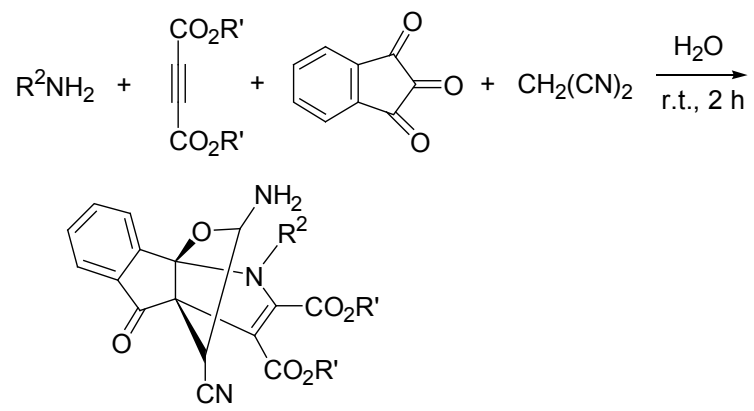

\section{2 丁炔二酸二甲酯参与的三组分反应}

在研究芳香胺、芳香醛和丁炔二酸酯的三组分反应 时, 发现了很有趣的实验现象: 在无催化剂存在下, 乙醇 中三组分反应生成了 2-羊基取代的四氢吡啶衍生物, 收 率在 $75 \%$ 以上，除强吸电子基团如硝基的芳香胺不能很 好反应外, 各种芳香醛和芳香胺都能够参加反应. 但是 改变反应条件, 在反应体系加入对甲苯磺酸作为催化剂, 该三组分反应则生成了 3-差基-2-吡咯酮衍生物, 收率中 等(Scheme 8). 在产物分子中引入羟基, 是非常有意义的 工作，该反应的机理还没有完全认识清楚，但是可以肯 定的是经过了 $\beta$-烯胺酯的生成和后续反应步骤 ${ }^{[29]}$.

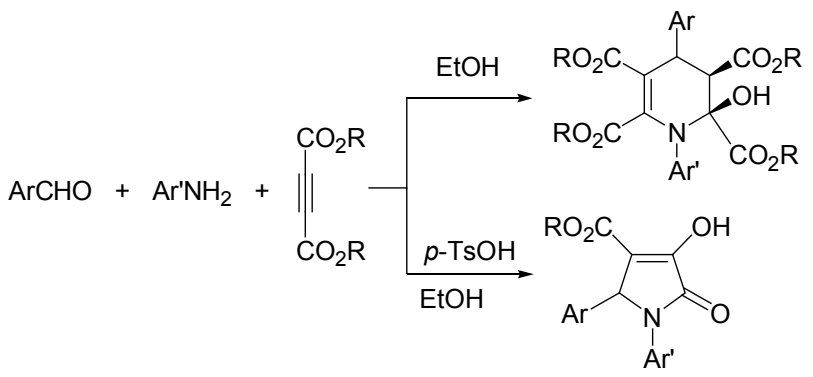

Scheme 8

江焕峰等 ${ }^{[30]}$ 的研究表明两分子芳香胺、芳香醛和丁 炔二酸酯在对甲苯磺酸催化下, 生成 3-芳胺基取代的 2吡咯酮衍生物. Khan 等 ${ }^{[31]}$ 报道伯胺、丁炔二酸二甲酯和
甲醛在分子碘催化下，也生成二氢吡咯烷酮衍生物. 江 焕峰等 ${ }^{[32]}$ 还采用苄胺和两分子丁炔二酸酯的三组分反 应在 $\mathrm{AgBF}_{4}$ 作为催化剂, PIDA 作为氧化剂的条件下, 生 成了五取代吡咯衍生物(Scheme 9).
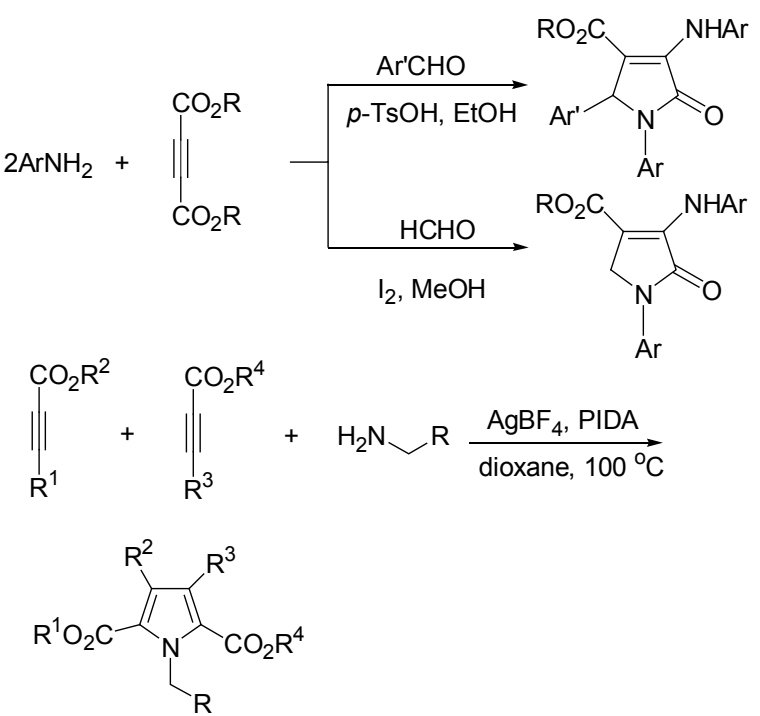

\section{Scheme 9}

Das 等 ${ }^{[33]}$ 报道了三氯化铁可以催化芳香胺、丁炔二 酸酯和溴代苯乙酮的三组分反应，制备了 $1,2,3,5$-四取 代吡咯衍生物. Nagarapu 等 ${ }^{[34]}$ 采用绿色化学合成方法, 以 PEG-400 作为反应介质，无需加入催化剂，该三组分 反应也可以顺利进行，得到高产率的四取代吡咯衍生 物, 脂肪胺也可以反应, 但产率普遍较低. Balalaie 等 ${ }^{[35]}$ 则报道了三氯化铁催化苠胺、丁炔二酸酯和硝基苯乙烯 的三组分反应，生成 1,2,3,4-四取代吡咯衍生物，在反应 中硝基作为离去基团被氨基取代(Scheme 10).

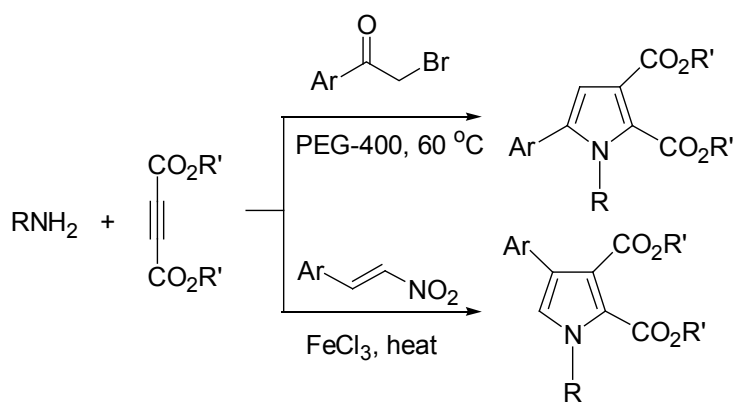

Scheme 10

Yavari 等 ${ }^{[36]}$ 用伯胺、异腈和两分子丁炔二酸酯在二 氯甲烷中反应，非常顺利地生成了 4-胺基-1,4-二氢吡啶 衍生物，收率在 $70 \%$ 以上(Scheme 11). 反应过程首先是 伯胺和异腈分别和丁炔二酸酯加成形成内盐和 $\beta$-烯胺 酯两个中间体，再经过质子转移、亲核加成和环合等一 系列反应完成的. 


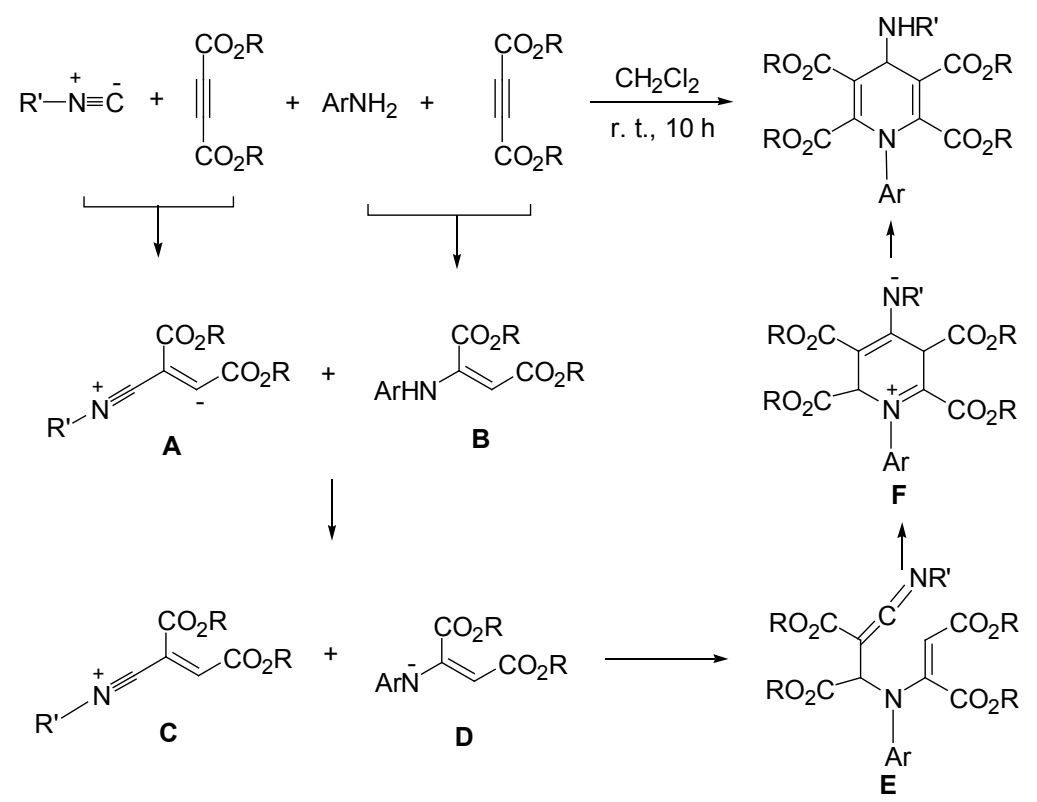

Scheme 11

该研究小组 ${ }^{[37]}$ 还报道了在 $N$-甲基咪唑催化下, 芳 香胺和两分子丁炔二酸酯反应, 生成吡定-2-酮衍生物, 条件温和, 收率在 $75 \%$ 以上(Scheme 12). 反应过程也是 按照 $N$-甲基咪唑、芳香胺分别与丁炔二酸酯形成 Huisgen 1,4-偶极子和 $\beta$-烯胺酯中间体, 然后两个中间 体间的质子转移和加成反应完成的. 在反应中, 如果先 让苄胺和一种丁炔二酸酯反应, 形成 $\beta$-烯胺酯中间体, 然后再加入 $N$-甲基咪唑和另一种丁炔二酸酯, 就可以 得到不同酯基的吡啶-2-酮衍生物. 但是, 如果在反应中 使用一分子丙炔酸酯和一分子丁炔二酸酯, 反应仅停留 在形成双 $\beta$-烯胺酯阶段, 不能完成环合反应. 这可能是 因为丙炔酸酯的反应活性较低所致.

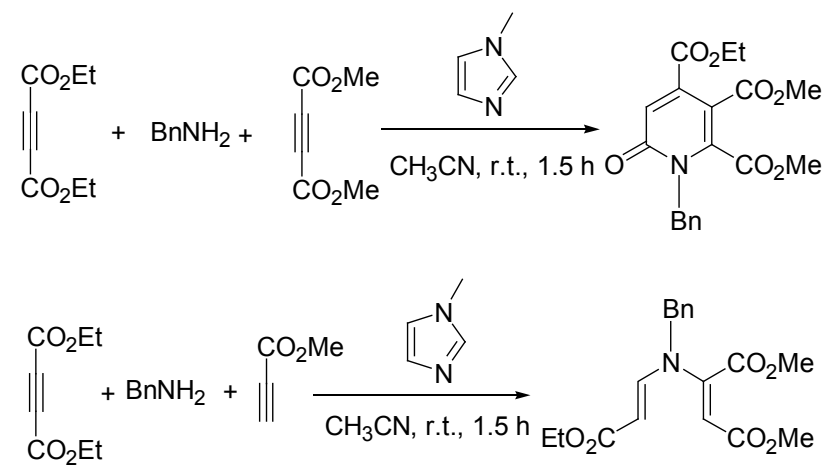

Scheme 12

Singh 等 ${ }^{[38]}$ 报道了 $L$-脯氨酸催化的 6 -氨基- $N, N^{\prime}$-二 甲基巴比妥酸、丁炔二酸酯和芳香醛的三组分反应, 得 到了吡啶并 $[2,3-d]$ 嘧啶衍生物(Eq. 10). 作者提出的反应 机理认为, 反应中并没有形成 $\beta$-烯胺酯中间体, 而是由 芳香醛先和氨基巴比妥酸缩合, 得到 6-亚氨基-5-芳亚
甲基- $N, N^{\prime}$-二甲基巴比妥酸, 后者具有 1-氮杂-1,3-丁二 烯结构单元, 可以和丁炔二酸酯进行 Diels-Alder 共扼加 成, 从而完成反应.

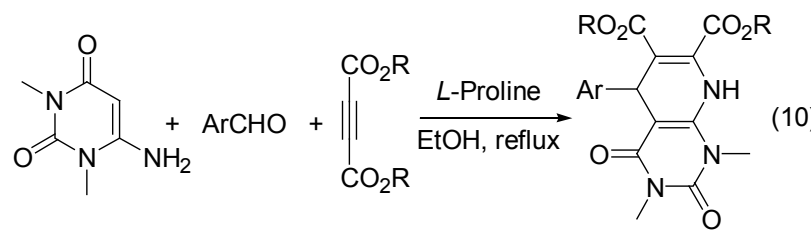

Khan 等 ${ }^{[39]}$ 报道了脂肪族伯胺、丁炔二酸酯和 2-丁 烯醛或者肉桂醛的三组分反应, 采用三氟乙酸作为催化 剂, 生成几乎等量的 1,4-二氢吡啶和 1,6-二氢吡啶衍生 物(Scheme 13). 如果采用三氟甲磺酸为催化剂, 反应具 有明显的选择性，1,4-二氢吡啶的收率可以达到 $70 \%$ 以 上. 作者认为之所以形成两种产物, 是由于现场形成的 $\beta$-烯胺酯可以和不饱和醛的醛基缩合形成亚胺中间体,

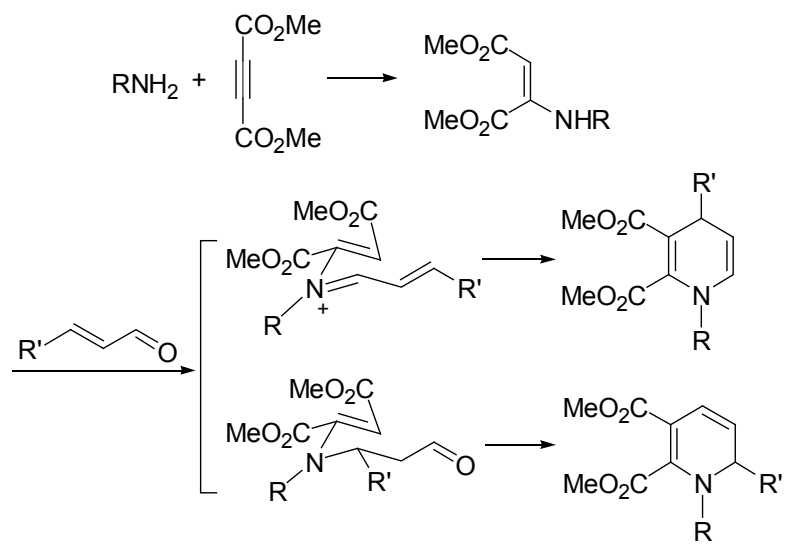

Scheme 13 
也可以进行 Michael 加成反应形成 $\beta$-胺基醛中间体，两 种中间体的后续环合分别得到 1,4-二氢吡啶和 1,6-二氢 吡啶衍生物. 有意思的是芳香胺不能进行此反应.

Teimouri 等 ${ }^{[40]}$ 研究了伯胺、丁炔二酸酯和 1,3 -二甲 基四氧嘧啶的三组分反应(Eq. 11). 在非常温和的条件 下，高产率地生成了螺环化合物，该反应可以使用多种 芳香族、脂肪族伯胺，甚至包含空间位阻很大的叔丁胺、 金刚烷基胺等. 反应过程包含 $\beta$-烯胺酯的形成和对羊基 的加成反应，以及后续的内酯化反应等串联反应过程.<smiles>[R]NC1=C(O[R])C(=O)C2(OC(=O)N(C)C(=O)N2C)C1=O</smiles>

Nageswar 等 ${ }^{[41]}$ 采用环糊精催化芳香胺、丁炔二酸 酯和 2,5-二甲氧基四氢呋喃在水中的三组分反应，高产 率地制备了 $N$-芳基氮杂环庚三烯-2,3-二甲酸酯. 王彦广 等 ${ }^{[42]}$ 使用二乙胺、环已胺等仲胺和丁炔二酸酯反应形成 的 $\beta$-烯胺酯, 在 Lewis 酸催化下, 与炔丙醇反应, 得到 二氢氮杂环庚三烯衍生物(Scheme 14).

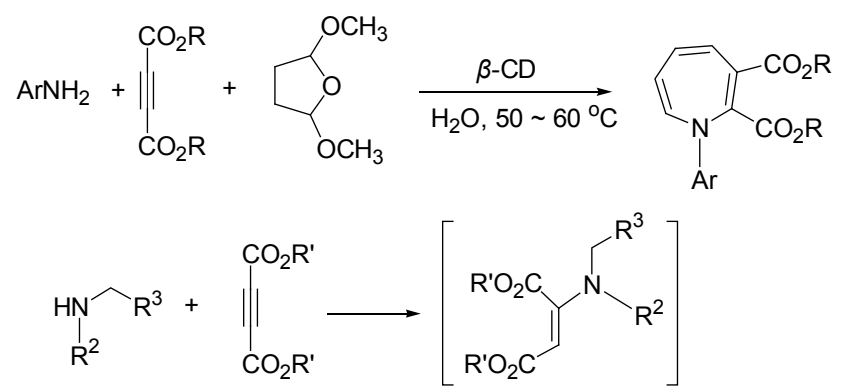<smiles>[R]OC(=O)C1=C([R])C([R])=CC([R])([R])C([R])N1[R]</smiles>

\section{Scheme 14}

屠树江等 ${ }^{[43]}$ 研究了芳基肼、芳香醛和丁炔二酸酯的 三分子反应，发现芳环上连有供电子基团的芳香醛得到 4-芳亚甲基吡唑-5-酮衍生物，而芳环上连有吸电子基团 的芳香醛形成芳亚甲基桥联的双吡唑-5-酮衍生物. 在 反应中芳基肼代替伯胺和丁炔二酸酯加成, 形成类似 $\beta$ 烯胺酯的芳肼基丁烯二酸酯中间体，后者再通过酯基的 氨解反应，形成吡唑-5-酤(Scheme 15).

Alizadeh 等 ${ }^{[44]}$ 报道了在三乙胺存在下苯肼、丁炔二 酸酯和芳酰氯的三组分反应，得到了 5-芳酰氧基吡唑衍 生物. 他们还报道通过芳基肼、丁炔二酸酯和狮三酮

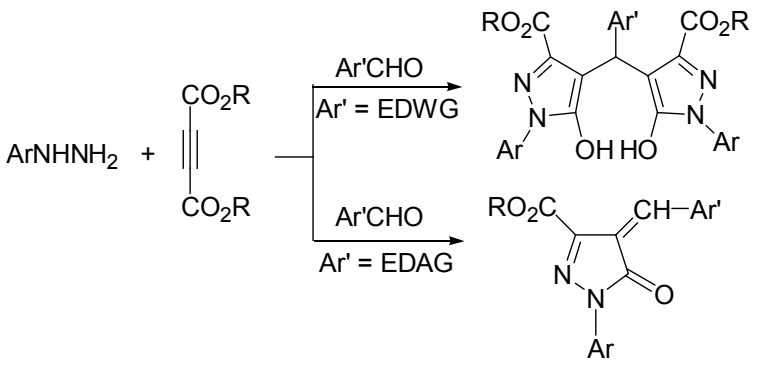

Scheme 15

的三组分反应，可以制备 4-狮三酩基取代的吡唑-5-酮 衍生物 ${ }^{[45]}$. 这些事例表明类似的含氮亲核试剂可以代 替胺和缺电子炔烃反应，形成活泼的中间产物，应用于 多组分串联反应(Scheme 16).

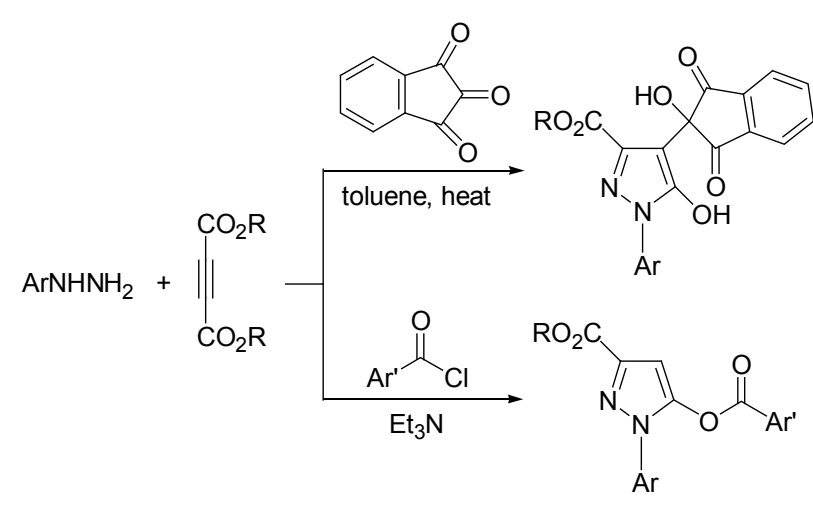

Scheme 16

\section{3 丙炔酸甲酯参与的多组分串联反应}

丙炔酸甲酯也是一种易得的缺电子烯烃，它可以和 芳香胺等亲核试剂反应，形成 $\beta$-烯胺酯，可能是因为缺 少一个吸电子的酯基，它和胺的加成反应速度比较慢， 同时丙炔酸甲酯不太稳定. 因此一般需要采用一锅煮的 操作方式，先将丙炔酸甲酯和芳香胺在乙醇等溶液中在 室温下反应过夜，然后再加入其它反应组分继续反应. 在反应中丙炔酸甲酯往往表现出不同于丁炔二酸二甲 酯的反应性质，得到明显不同的产物.

Sirijindalert 等 ${ }^{[46]}$ 报道脂肪族或者芳香族伯胺和丙 炔酸甲酯在二氯甲烷中加成反应，在温和的条件下就可 以形成 $\beta$-烯胺酯. 后者在四氯化钛催化下, 经过一系列 串联反应，生成 1,4-二氢吡啶衍生物(Scheme 17).

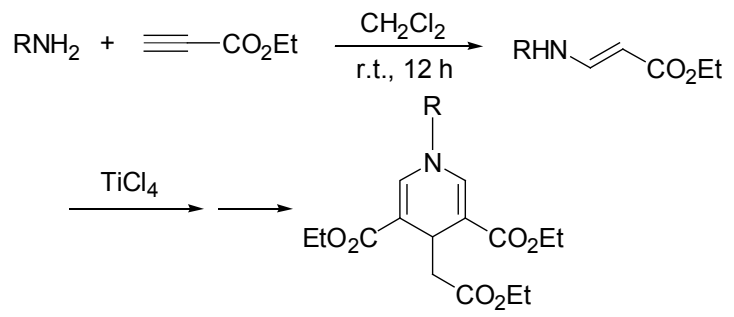

Scheme 17 
如将由丙炔酸甲酯和芳香胺在乙醇中生成的 $\beta$-烯 胺酯再和芳香醛、丙二腈在三乙胺存在下进行反应, 顺 利得到预期的 1,4-二氢吡啶衍生物. 用氧乙酰胺代替丙 二腈进行反应，也生成含有酰胺官能团的 1,4-二氢吡啶 衍生物. 在相同情况下, 氧乙酸乙酯的反应情况有很大 不同, 仅在个别情况下生成少量预期的酯基取代的 1,4二氢吡啶衍生物, 主要生成的是吡啶-2-酮衍生物, 在反 应过程中, 氧基未参加反应, 反而是酯基被氨解, 得到 最后产物(Scheme 18) ${ }^{[47]}$.

Kikuchi 等 ${ }^{[48]}$ 报道了在三氟甲磺酸锌催化下, 丙炔 酸乙酯和亚胺反应, 生成 1,4-二氢吡啶衍生物, 在反应 过程中亚胺明显分解成了醛和胺两个组分, 再通过丙炔 酸乙酯和胺的加成反应, 形成 $\beta$-烯胺酯中间体进而完 成反应(Eq. 12).<smiles>[R]C=NN=[Z7]C(=O)OCCOc1ccccc1C(=O)OCC</smiles>

进一步的研究发现, 由丙炔酸甲酯和芳香胺在乙醇 中生成的 $\beta$-烯胺酯, 继续和芳香醛、氭基频哪酮在三乙 胺中反应, 得到了完全不同的实验结果 ${ }^{[49]}$. 反应生成的 是 2,3-双取代的丙烯酸甲酯衍生物, 并且存在 $Z, E$-异构 体, 其中以 $Z$-异构体为主, $Z / E>4: 1$. 产物的顺反异构 来源于生成的 $\beta$-烯胺酯中间体存在的顺反两种构型 (Scheme 19). 整个反应停留在 $\beta$-烯胺酯对现场形成的芳 亚甲基氰基频哪酮的加成阶段, 未能再进一步通过氨基
对氧基的加成形成环状化合物. 试图通过提高反应温度 等措施促进环合, 并没有取得成功, 反而导致产物的分 解. 在类似条件下, $\beta$-烯胺酯和芳香醛、环戊二酮的串联 反应可以生成相应的多取代丙烯酸甲酯衍生物, 并且基 本都得到 Z-异构体. 利用预先制备好的硝基苯乙烯类化 合物和 $\beta$-烯胺酯反应也主要生成 $Z$-构型的丙烯酸甲酯 衍生物 ${ }^{[50]}$.

Teimouri 等 ${ }^{[51]}$ 也报道了伯胺、丙炔酸酯和 1,3 -烷基 四氧嘧啶的三组分反应，在水中于室温下快速生成了高 产率的巴比妥酸连接的胺基丙烯酸衍生物, 反应无疑是 通过胺和丙炔酸酯加成形成的 $\beta$-烯胺酯中间体，对羰基 的加成反应完成的(Eq. 13). 如果使用的是丁炔二酸酯, 在此三组分反应中则表现出明显不同的反应性质 (Scheme 12).

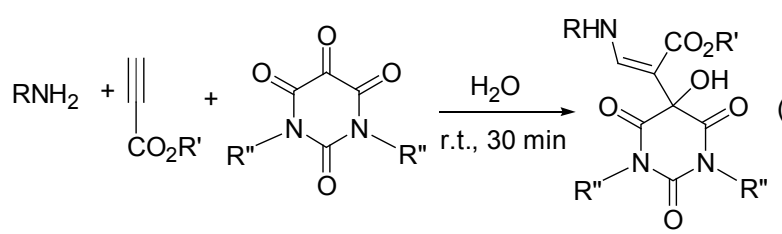

综上所述, 由缺电子炔烃和胺现场生成的 $\beta$-烯胺酯 具有非常丰富的化学反应性质, 可以参与多种类型的多 组分串联反应，为一些结构复杂的五元或六元杂环化合 物提供了新的简捷合成方法. 该领域虽然目前已经取得 了一些有价值的研究成果, 但对于缺电子炔烃和胺直接 原位生成的 $\beta$-烯胺酯参加的多组分反应的设计和应用 还有很大的发展潜力, 今后将进一步拓展直接原位生成

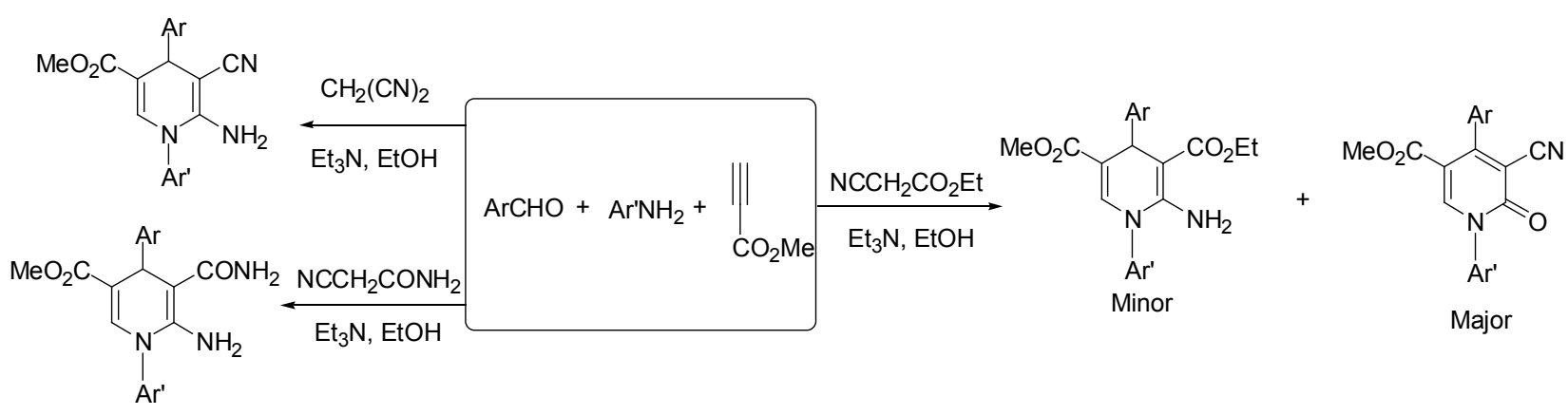

Scheme 18

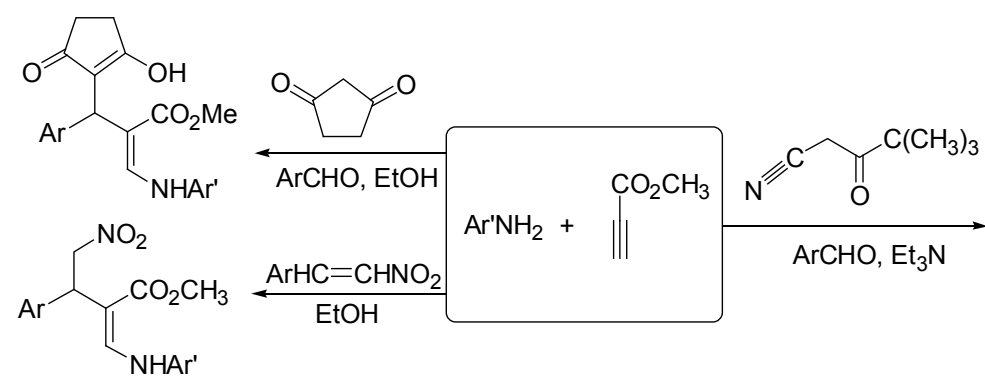<smiles>COC(=O)NC=C(C(=O)O)C(C#N)C(C#N)C(=O)OC</smiles>

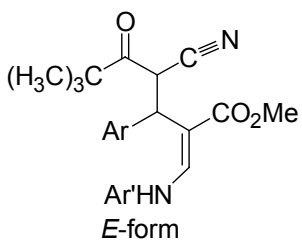

Scheme 19 
的 $\beta$-烯胺酯的结构多样性和反应性能, 使其成为一种有 广泛应用价值的有机合成新方法.

\section{References}

[1] (a) Huisgen, R.; Morikawa, M.; Herbig, K.; Brunn, E. Chem. Ber. 1967, 100, 1094. (b) Huisgen, R. Z. Chem. 1968, 8, 290

[2] Nair, V.; Rajesh, C.; Vinod, A. U.; Bindu, S.; Sreekanth, A. R.; Mathen, J. S.; Balagopal, L. Acc. Chem. Res. 2003, 36, 899

[3] Nair, V.; Menon, R. S.; Sreekanth, A.; Abhilash, N.; Biju, A. T. Acc. Chem. Res. 2006, 39, 520.

[4] Yavari, I.; Mirzaei, A.; Moradi, L.; Khalili, G. Tetrahedron Lett. 2010, 51, 396

[5] Tejedor, D.; López-Tosco, S.; Cruz-Acosta, F.; Méndez-Abt, G.; García-Tellado, F. Angew. Chem., Int. Ed. 2009, 48, 2090.

[6] Dai, L. Z.; Qi, M. J.; Shi, Y. L.; Liu, X. G.; Shi, M. Org. Lett. 2007, 9, 3191.

[7] Wang, Y. G.; Cui, S. L.; Lin, X. F. Org. Lett. 2006, 8, 1241.

[8] (a) Cho, C. S. Tetrahedron Lett. 2005, 46, 1415.

(b) Ziyaei-Halimehjani, A.; Saidi, M. R. Tetrahedron Lett. 2008, 49, 1244.

[9] Zhu, Q. H.; Jiang, H. F.; Li, J. H.; Zhang, M.; Wang, X. J.; Qi, C. R. Tetrahedron 2009, 65, 4604.

[10] (a) Lue, P.; Greenhill, J. V. Adv. Heterocycl. Chem. 1997, 67, 215. (b) Elassara, A. Z.; El-Khair, A. A. Tetrahedron 2003, 59, 8463.

[11] (a) Katritzky, A. R.; Hayden, A. E.; Kirichenko, K.; Pelphrey, P.; Ji, Y. J. Org. Chem. 2004, 69, 5108.

(b) Sirijindalert, H.; Hansuthirakul, K.; Rashatasakhon, P.; Sukwattanasinitt, M.; Ajavakom, A. Tetrahedron 2010, 66, 5161.

[12] (a) Vohra, R. K.; Bruneau, C.; Renaud, J. Adv. Synth. Catal. 2006, $348,2571$.

(b) Kumar, A.; Maurya, R. A. Tetrahedron 2008, 64, 3477.

[13] (a) Arcadi, A.; Bianchi, G.; Di Giuseppe, S.; Marinelli, F. Green Chem. 2003, 64.

(b) Khosropour, A. R.; Khodaei, M. M.; Kookhazadeh, M. Tetrahedron Lett. 2004, 45, 1725.

[14] (a) Liu, W. B.; Jiang, H. F.; Huang, L. B. Org. Lett. 2010, 12, 312. (b) Liu, W. B.; Jiang, H. F.; Zhang, M.; Qi, C. R. J. Org. Chem. 2010, $75,966$.

(c) Bayat, M.; Imanieh, H.; Hassanzadeh, F. Tetrahedron Lett. 2010, 51,1873 .

(d) Terzidis, M. A.; Stephanidou-Stephanatou, J.; Tsoleridis, C. A.; Terzis, A.; Raptopoulou, C. P.; Psycharis, V. Tetrahedron 2010, 66, 947.

[15] (a) Yavari, I.; Mirzaei, A.; Moradi, L.; Khalili, G. Tetrahedron Lett. 2010, 51, 396

(b) Yavari, I.; Seyfi, S.; Hossaini, Z. Tetrahedron Lett. 2010, 51, 2193.

(c) Kumaraswamy, G.; Rambabu, D.; Jayaprakash, N.; Rao, G. V.; Sridhar, B. Eur. J. Org. Chem. 2009, 4158.

[16] (a) Alizadeh, A.; Rostamnia, S.; Hu, M. L. Synlett 2006, 1592.

(b) Adib, M.; Mohammadi, B.; Mahdavi, M.; Abbasi, A.; Kesheh, M. R. Synlett 2007, 2497.

(c) Alizadeh, A.; Rostamnia, S.; Zhu, L. G. Tetrahedron Lett. 2010 , 51,4750 .

(d) Teimouri, M. B.; Abbasi, T. Tetrahedron 2010, 66, 3795.

[17] (a) Kielland, N.; Lavilla, R. Top. Heterocycl. Chem. 2010, 25, 127.

(b) Shaabani, A.; Rezayan, A. H.; Sarvary, A. Mol. Divers. 2011,
15,41

[18] Sun, J.; Xia, E. Y.; Wu, Q.; Yan, C. G. Org. Lett. 2010, 12, 3678.

[19] Yamuna, E.; Zeller, M.; Prasad, K. J. R. Tetrahedron Lett. 2011, 52,6805 .

[20] Lei, M.; Zhan, Z. J.; Tian, W.; Lu, P. Tetrahedron 2012, 68, 3361.

[21] Zhang, M.; Jiang, H. F.; Liu, H. L.; Zhu, Q. H. Org. Lett. 2007, 9, 4111

[22] Sun, J.; Xia, E. Y.; Wu, Q.; Yan, C. G. ACS Comb. Sci. 2011, 13, 421.

[23] Sun, J.; Wu, Q.; Xia, E. Y.; Yan, C. G. Eur. J. Org. Chem. 2011 2981.

[24] Sun, J.; Sun, Y.; Gao, H.; Yan, C. G. Eur. J. Org. Chem. 2012, 1976.

[25] Sun. J.; Wu, Q.; Zhang, L. J.; Yan, C. G. Chin. J. Chem. 2012, 30, 1548.

[26] Kiruthika, S. E.; Lakshmi, N. V.; Banu, B. R.; Perumal, P. T. Tetrahedron Lett. 2011, 52, 6508.

[27] Sun, Y.; Sun, J.; Yan, C. G. Tetrahedron Lett. 2012, 53, 3647.

[28] Alizadeh, A.; Rezvanian, A.; Zhu, L. G. J. Org. Chem. 2012, 77, 4385 .

[29] Sun, J.; Sun, Y.; Gao, H.; Yan, C. G. Eur. J. Org. Chem. 2011, 6952.

[30] Zhu, Q.; Jiang, H. F.; Li, J.; Liu, S.; Xia, C.; Zhang, M. J. Comb. Chem. 2009, 11, 685.

[31] Khan, A. T.; Ghosh, A.; Khan, A. M. Tetrahedron Lett. 2012, 53, 2622.

[32] Liu, W. B.; Jiang, H. F.; Huang, L. B. Org. Lett. 2010, 12, 312.

[33] Das, B.; Chinna Reddy, G.; Balasubramanyam, P.; Aneyulu, V. Synthesis 2010, 1625.

[34] Nagarapu, L.; Mallepalli, R.; Yeramanchi, L.; Bantu, R. Tetrahedron Lett. 2011, 52, 3401.

[35] Ghabraie, E.; Balalaie, S.; Bararjanian, M.; Bijanzadeh, H. R.; Rominger, F. Tetrahedron 2011, 67, 5415.

[36] Yavari, I.; Bayat, M. J.; Sirouspour, M.; Souri, S. Tetrahedron 2010, 66, 7995.

[37] Yavari, I.; Bayat, M. J. Tetrahedron Lett. 2011, 52, 6649.

[38] Samai, S.; Nandi, G. C.; Chowdhury, S.; Singh, M. S. Tetrahedron 2011, 67, 5935.

[39] Khan, A. T.; Khan, M. M. Tetrahedron Lett. 2011, 52, 3455.

[40] Teimouri, M. B.; Abbasi, T. Tetrahedron 2010, 66, 3795.

[41] Ramesh, K.; Murthy, S. N.; Nageswar, Y. V. D. Tetrahedron Lett. 2011, 52, 2362.

[42] Yin, G. W.; Zhu, Y. X.; Lu, P.; Wang, Y. G. J. Org. Chem. 2011, 76, 8922.

[43] Tu, X. C.; Feng, H.; Tu, M S.; Jiang, B.; Wang, S. L.; Tu, S. J. Tetrahedron Lett. 2012, 53, 3169.

[44] Zohreh, N.; Alizadeh, A. Tetrahedron 2011, 67, 4595.

[45] Alizadeh, A.; Zarei, A.; Alizadeh, A. R. A.; Zarei, A.; Rezvanian, A. Helv. Chim. Acta 2012, 95, 278.

[46] Sirijindalert, T.; Hansuthirakul, K.; Rashatasakhon, P.; Sukwattanasinitt, M.; Ajavakom, A. Tetrahedron Lett. 2011, 52, 5789.

[47] Sun, J.; Sun, Y.; Xia, E. Y.; Yan, C. G. ACS Comb. Sci. 2011, 13, 436.

[48] Kikuchi, S.; Iwai, M.; Murayama, H.; Fukuzawa, S. Tetrahedron Lett. 2008, 49, 114

[49] Sun, J.; Sun, Y.; Gao, H.; Yan, C. G. Synthesis 2012, 1069.

[50] Sun, Y.; Sun, J.; Yan, C. G. Mol. Divers. 2012, 16, 163.

[51] Teimouri, M. B.; Abbasi, T.; Mivehchi, H. Tetrahedron 2008, 64, 10425 . 